\title{
Revision Patellar Tendon Reconstruction in Prosthetic Joint Infection following Total Knee Arthroplasty - A Case Report
}

\author{
Vipin Mohan ${ }^{\mathrm{a}}$, Jai Thilak ${ }^{\mathrm{a}}$, Nayanika Suresh ${ }^{\mathrm{a}}$ \\ a. Department of Orthopaedics, Amrita Institute of Medical Sciences, Kochi, Kerala, India
}

Published on $1^{\text {st }}$ June 2020

\begin{abstract}
Patellar tendon rupture is a rare but devastating complication following total knee arthroplasty. We report a case of a failed patellar tendon reconstruction and prosthetic joint infection following patellar tendon rupture. The objective of this case report is to highlight the favorable outcome following revision patellar tendon reconstruction with a synthetic graft and two stage revision arthroplasty.
\end{abstract}

Key Words: Total Knee Revision Patellar Tendon

\section{INTRODUCTION}

$\mathrm{P}$ atellar tendon rupture is a serious complication following total knee arthroplasty (TKA). Its occurrence ranges from $0.17 \%$ to $1 \% .^{1-4}$

The various treatment options ranges from direct repair of the ruptured tendon to reconstruction using autologous tendons or muscles and usage of various allografts and implantation of synthetic grafts. ${ }^{5}$

This is the case of a patient with Rheumatoid arthritis (RA) on disease modifying drugs (DMARDs) who developed atraumatic rupture of patellar tendon following TKA, which was managed by reconstruction with autogenous semitendinosus graft. The patient went on to develop prosthetic joint infection (PJI) along with attenuation of the reconstructed patella tendon, which was managed by revision patellar tendon reconstruction and two staged revision TKA.

Although there is a good amount of literature exists on various techniques of management of patellar tendon rupture following TKA. There is limited literature present on the management of the PJI following patellar tendon reconstruction. A recent review suggests knee arthrodesis in the cases of chronic rupture or failure of previous treatment or multiple surgical procedures on the knee with the evidence of sepsis ${ }^{5}$. Other published case series of patella tendon reconstructions with associated infection also reports inferior outcome with reconstructions with complications including recurrent infections, need for arthrodesis or significant extensor lag. ${ }^{6,7}$

This case report outlines a case of failed patella tendon reconstruction developing prosthetic joint infection (PJI) following TKA and its management and successful outcome up to three years of follow up.

\section{CASE PRESENTATION}

Our patient is a 45-year-old Indian woman who is a known case of RA on treatment with DMARDs for the past 15 years. She underwent simultaneous bilateral total knee arthroplasty (TKA) three years ago at an outside facility. One month following the surgery she developed pain on the left knee as well as difficulty in walking. She was diagnosed to have rupture of the patellar tendon and she underwent patellar tendon reconstruction with autogenous semitendinosus graft by her primary surgeon and the knee was immobilized in extension for six weeks. Three months following the patellar tendon reconstruction she developed prosthetic joint infection on the same knee with wound dehiscence and underwent arthrotomy, debridement with

Cite this article as: Mohan V, Thilak J, Suresh N. Revision Patellar Tendon Reconstruction in Prosthetic Joint Infection following Total Knee Arthroplasty - A Case Report. KJOrth. 2020 Jun 1;33(01):34-7. 


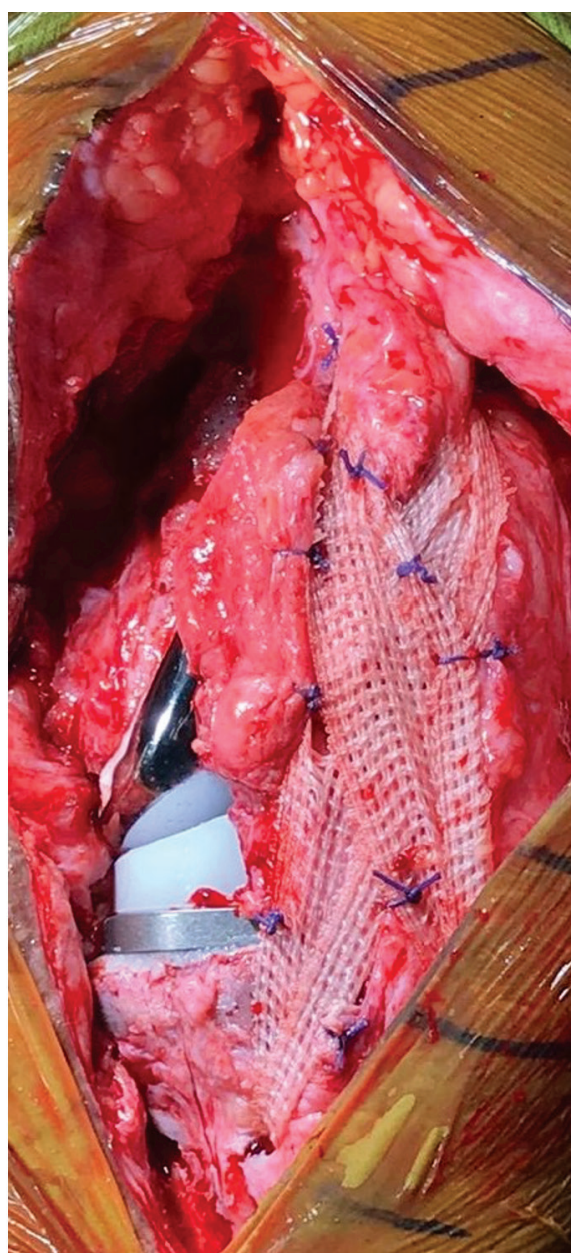

Figure 1A. Synthetic Allograft tendon augmentation

fasciocutaneous flap-cover procedure and retention of the prosthesis. Thereafter, she was walking with the support of a walker. Five months following the arthrotomy and debridement she was referred to our institute by the primary surgeon with the suggestion of knee arthrodesis as since the patient had recurrence of infection as well as inability to actively extend the knee indicating failure of the patellar tendon reconstruction. She presented to us with a history of clear discharge from the surgical scar since the past two months, associated with swelling and pain over the left knee and discomfort while walking. The symptoms where progressively worsening. She denied any history of trauma, fever or recent systemic infection.

Upon presentation to us, her left lower extremity revealed a discharging sinus over the distal third of the midline surgical scar; discharge was clear and there was associated knee effusion. Surrounding skin was erythematous with diffuse tenderness. A healthy transposed fasciocutaneous flap from lateral side of left knee was observed on the lateral side. There was significant quadriceps muscle wasting. The joint was stable, but joint movement was restricted to $5^{0}$ to $30^{\circ}$ with extensor lag of $10^{\circ}$.

Laboratory investigations revealed elevated Inflammatory markers - C-reactive protein was $72 \mathrm{mg} / 1$ (normal $<1 \mathrm{mg} / \mathrm{l}$ )

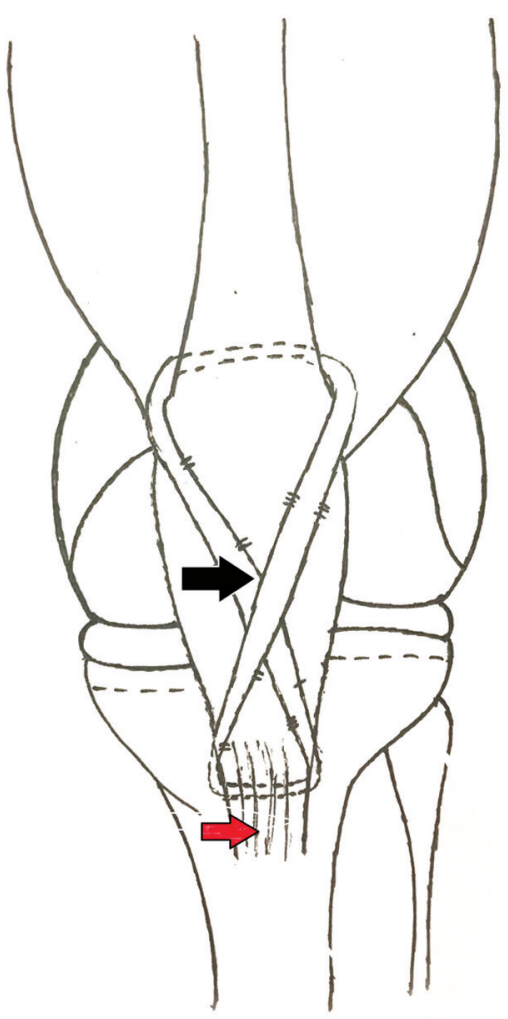

Figure 1B. The Synthetic ligament reconstruction pattern over the attenuated ligamentum patellae

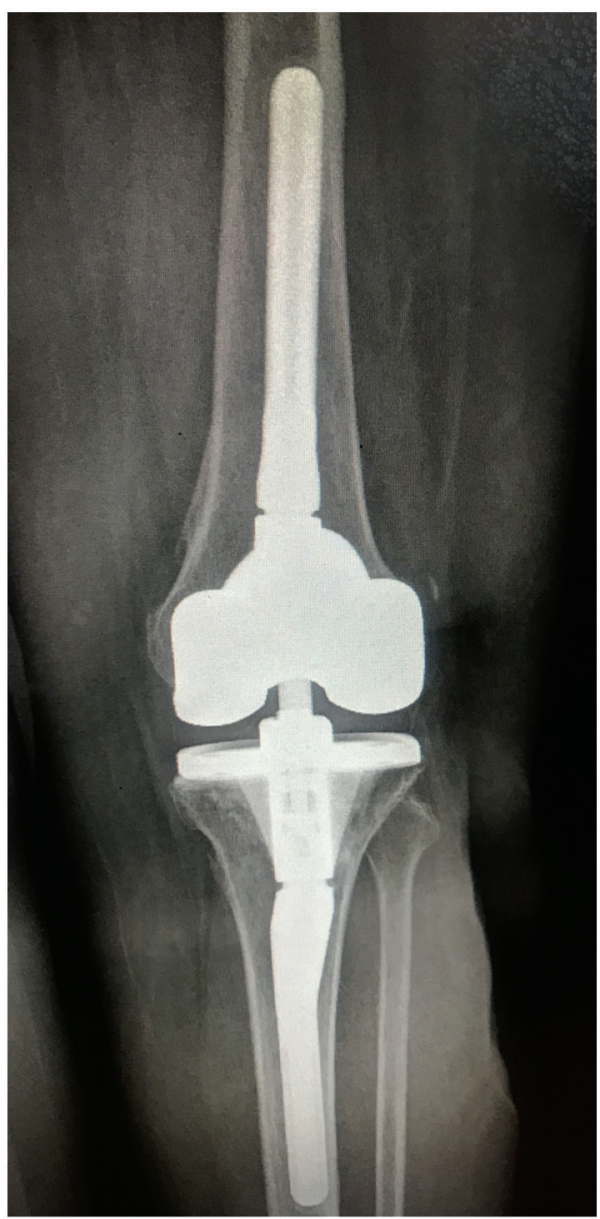

Figure 2A. Revision TKA Radiograph AP view 


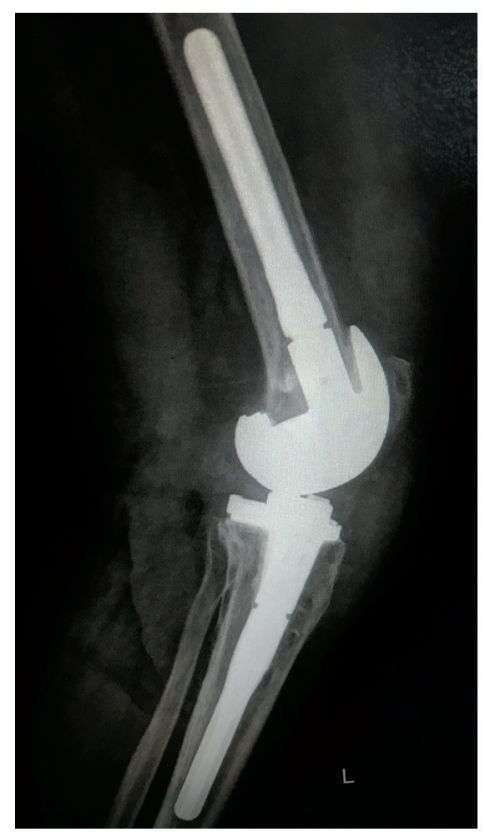

Figure 2B. Revision TKA Radiograph Lateral view

and erythrocyte sedimentation rate was $81 \mathrm{~mm} / \mathrm{hr}$ (normal $<20 \mathrm{~mm} / \mathrm{hr}$ ). Arthrocentesis of the left knee showed a white-blood cell count of 50000 cells $/ \mu \mathrm{L}$ (normal $<3000$ cells $/ \mu \mathrm{L}$ ), and culture of aspirated synovial fluid revealed growth of Methicillin resistant Staphylococcus aureus (MRSA). The presence of the sinus tract communicating with the joint was sufficient to make the definite diagnosis of prosthetic joint infection as it meets the criterion laid down by the Musculoskeletal Infection Society. ${ }^{8}$ Also, the synovial fluid culture yielded the growth of a pathogen (MRSA) supplemented the diagnosis of PJI.

She underwent Stage I surgery - sinus tract which was noted to extend inferior to the joint was excised and extraction of the prosthesis was carried out. Intra-operatively calcium sulphate beads (Stimulan - Biocomposites Ltd, Keele, UK) mixed with Vancomycin powder (2 grams $/ 10 \mathrm{cc}$ ) was applied. Intra operatively it was noted that the reconstructed patella tendon was attenuated. Tissue samples collected during the surgery also grew Methicillin Resistant Staphylococcus aureus on cultures. Post - operatively she continued to receive intravenous Vancomycin along with oral Rifampicin in consultation with the clinical microbiologist. Her immediate postoperative period was uneventful. Serial labs showed a downward trend in inflammatory markers and cell counts. Sutures were removed in Post-operative day 15. Postoperatively she received antibiotics for 6 weeks.

Two weeks after completing six weeks of antibiotic treatment an arthrocentesis of Left knee was done which revealed a sterile culture.

Three months following the stage I surgery, she was re-admitted for Stage II revision TKA and revision patellar tendon reconstruction under Spinal Anesthesia. Intraoperatively the previously reconstructed patella tendon

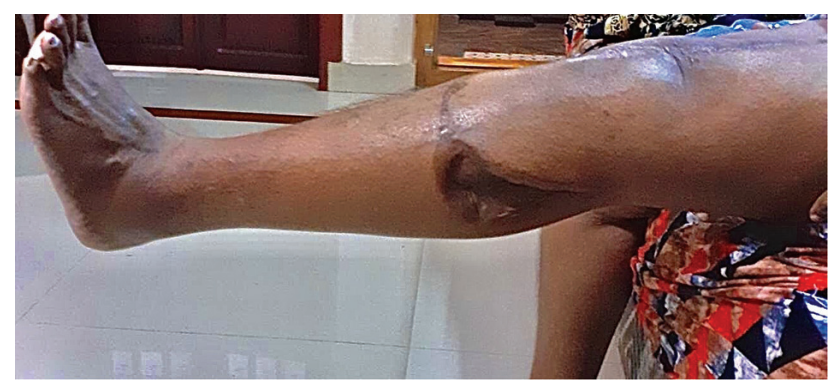

Figure 3A. Range of motion extension without lag

was found to be in an attenuated state. She underwent re-implantation with Revision implants (Nexgen CCK; Zimmer, Warsaw, Indiana) along with patellar tendon tissue augmentation using a synthetic allograft - open weave poly-tape implant (Patellar Tape System ${ }^{\mathrm{TM}}-\mathrm{Neol}-$ igaments, Springfield house, Leeds, UK) (Figure 1A \& B)

Fresh soft tissue cultures showed no growth. She was put on a motion limiting knee brace locked at $0-40$ degrees of flexion post surgery with a plan to gradually increase the range of movement over the next 3 months. Patient was discharged with the help of a walking aid.

In succeeding review visits over a span of three years, patient was able to mobilize well and eventually walk comfortably without support, serial laboratory investigations showed settling inflammatory markers and radiographs revealed satisfactory implant position and no loosening of prosthesis at the end of three years (Figure 2A \& B)

At the latest follow up which is 3 years following the stage II revision surgery she is walking independently without pain or instability. On clinical examination knee joint showed $0^{0}-95^{\circ}$ range of motion and no extensor lag (Figure 3 A \& B)

Quadriceps power was found be MRC grade 5. Knee society scoring (KSS), knee score for the knee was found to be 82 and functional score was found to be 78 .

\section{DISCUSSION}

Patellar tendon rupture is a rare but serious complication of total knee arthroplasty (TKA). The incidence of

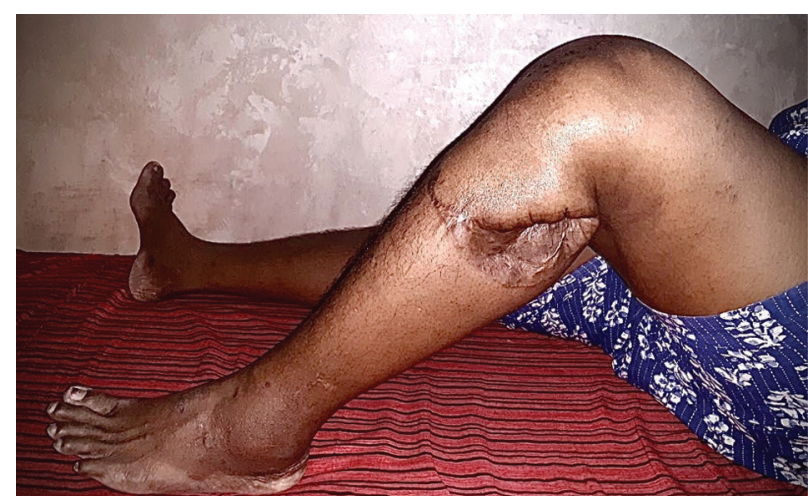

Figure 3B. Range of movement showing flexion 
this complication has been reported to be from $0.17 \%$ to $1 \% .^{1-4}$ The surgical techniques can be classified into five categories such as simple repair, repair with augmentation by a neighboring tendon, reconstruction using an artificial ligament, replacement by an allograft and salvage techniques. $^{5}$

One common complication following patella tendon reconstruction is deep infection. Rand et $\mathrm{al}^{2}$ reported that infection developed in 4 out of 17 patients treated for patella tendon ruptures following TKA.

Staphylococcus aureus is the most common causative pathogen for PJI in RA patients, which is similar to that in patients without RA., methicillin-resistant Staph aureus can be the causative organism in as high as $35 \%$ of the cases of PJI in RA patients. ${ }^{9}$

There are only a few literature evidences available for multiple surgeries for the reconstruction of the patellar tendon with associated Prosthetic joint infection (PJI). A recent review suggests knee arthrodesis in the case of chronic rupture or failure of previous treatment or multiple surgical procedures on the knee with the evidence of sepsis. $^{5}$

A series of 3 cases reported by Wilson et $\mathrm{al}^{6}$ stated the need of multiple surgeries to control the infection and stabilize the knee. In their series, one of the three patients continued to have residual infection. The combination of patella tendon rupture along with deep infection resulted in a poor functional outcome even after reconstruction.

Another report of 2 cases by Aikawa et $\mathrm{al}^{7}$ reported the need for arthrodesis following bilateral prosthetic joint infection (PJI) with MRSA after synthetic grafting procedure for one knee and staged revision surgery on the other knee after control of infection. Another case of infection with Staphylococcus epidermidis after reconstruction of her patellar tendon by a synthetic graft procedure. A staged revision TKA and a tendon reconstruction by BTB allograft was performed but the patient had an extension lag of $30^{\circ}$.

Our case is that of patient who had undergone TKA for inflammatory arthritis (RA) with atraumatic rupture of patellar tendon post-operatively. Following reconstruction of the patellar tendon she developed prosthetic joint infection along with attenuation of the reconstructed patellar tendon. The infection recurred after the debridement with retention of prosthesis. Later on, 2 staged revision TKA along with revision reconstruction with synthetic graft was performed. The virulence of the pathogen (MRSA) as well as the inflammatory arthritis (RA) posed a unique challenge in the management of the condition. At the latest follow up after 3 years following the revision surgery she is found to have good outcome.

\section{CONCLUSION}

Patellar tendon rupture with prosthetic joint infections are disastrous complications following TKA. This is a case of infected TKA with incompetent Ligamentum patellae, treated by a two-stage revision and synthetic graft reconstruction of the patellar ligament resulted in a good outcome.

\section{END NOTE}

\section{Author Information}

1. Dr Vipin Mohan, MS (Ortho), Assistant Professor, Department of Orthopaedics, Amrita Institute of Medical Sciences, Kochi, Kerala, India

2. Dr Jai Thilak, MS, MCh (UK), FRCS (Glasg), Clinical Professor, Department of Orthopaedics, Amrita Institute of Medical Sciences, Kochi, Kerala, India

3. Dr Nayanika Suresh, MBBS, House surgeon, Department of Orthopaedics, Amrita Institute of Medical Sciences, Kochi, Kerala, India

Conflict of Interest: None declared

\section{REFERENCES}

1. Schoderbek RJ, Brown TE, Mulhall KJ, Mounasamy V, Iorio R, Krackow KA, et al. Extensor mechanism disruption after total knee arthroplasty. Clin Orthop Relat Res. 2006 May;446:176-85.

2. Rand JA, Morrey BF, Bryan RS. Patellar tendon rupture after total knee arthroplasty. Clin Orthop Relat Res. 1989 Jul;(244):233-8.

3. Nam D, Abdel MP, Cross MB, LaMont LE, Reinhardt KR, McArthur BA, et al. The management of extensor mechanism complications in total knee arthroplasty. AAOS exhibit selection. J Bone Joint Surg Am. 2014 Mar 19;96(6):e47.

4. Papalia R, Vasta S, D’Adamio S, Albo E, Maffulli N, Denaro V. Complications involving the extensor mechanism after total knee arthroplasty. Knee Surg Sports Traumatol Arthrosc. 2015 Dec;23(12):3501-15.

5. Bonnin M, Lustig S, Huten D. Extensor tendon ruptures after total knee arthroplasty. Orthop Traumatol Surg Res. 2016 Feb;102(1 Suppl):S21-31.

6. Wilson K, Kothwal R, Khan WS, Williams R, Morgan-Jones R. Patella tendon injuries secondary to cement spacers used at first-stage revision of infected total knee replacement. Front Surg. 2015;2:11.

7. Aikawa J. Salvage Surgery for Postoperative Infection Related to Patellar Tendon Reconstruction Following Total Knee Arthroplasty: A Report of Two Cases. Japanese Journal of Joint Diseases. 2014;33(2):189-94.

8. Parvizi J, Tan TL, Goswami K, Higuera C, Della Valle C, Chen AF, et al. The 2018 Definition of Periprosthetic Hip and Knee Infection: An Evidence-Based and Validated Criteria. J Arthroplasty. 2018 May;33(5):1309-1314.e2.

9. Hsieh PH, Huang KC, Shih HN. Prosthetic joint infection in patients with rheumatoid arthritis: an outcome analysis compared with controls. PLoS One. 2013;8(8):e71666. 